\title{
Uncovering the influence of diet and gut microbiota in human serum metabolome
}

\section{David Obeso $^{1,2}$ | Elisa Zubeldia-Varela ${ }^{1,2}$ | Alma Villaseñor ${ }^{2}$ (C)}

${ }^{1}$ Facultad de Farmacia, Centro de Metabolómica y Bioanálisis (CEMBIO, Universidad San Pablo-CEU, CEU Universities, Urbanización Montepríncipe, Madrid, España

${ }^{2}$ Facultad de Medicina, Departamento de Ciencias Médicas Básicas, Instituto de Medicina Molecular Aplicada (IMMA, Universidad San Pablo-CEU, CEU Universities, Madrid, España

Correspondence: Alma Villaseñor, Instituto de Medicina Molecular Aplicada (IMMA), Departamento de Ciencias Médicas Básicas. Facultad de Medicina. Universidad San Pablo-CEU, CEU Universities, Madrid, España.

Email: alma.villasenor@ceu.es

Funding information:

This work was supported by Instituto de Salud Carlos III (Project number PI19/00044) co-funded by the European Regional Development Fund "Investing in your future" for the thematic network and co-operative research centers ARADyAL RD16/0006/0015. A.V. is funded by a postdoctoral research fellowship from ARADYAL and D.O. by the European project DIFAMEM (PCI2018-092930).

Keywords: allergy, diet, metabolome, metabolomics, microbiota, serum

\section{1 | INTRODUCTION}

Metabolomics is the science focused on the study of the metabolism in living organisms. ${ }^{1}$ Their aim is to measure the metabolites that are the intermediate and final molecules of the biological processes. The entire collection of the metabolites is called the metabolome. Changes in the biological system would be reflected in the metabolome, since metabolites are involved in the biological functions. The metabolome is affected by the interaction of different factors such as the host, diet, lifestyle, pharmacological treatment, and gut microbiota among others. Being the latter, one of the most important factors as the gut hosts the largest number of microorganisms in the body (eg, up to $10^{9}$ bacteria per gram of luminal content ${ }^{2}$ ).

Currently, there is not a single technique that detects the entire metabolome. Metabolomics uses high-throughput techniques that allow structural elucidation, such as mass spectrometry (MS), which in combination with a separation technique such as liquid chromatography (LC) provides broad detection of metabolites.

Recently, Bar et al analyzed 491 serum samples from healthy individuals using LC-MS, for whom they have collected extensive clinical lifestyle dietary, genetics, and gut microbiota data. ${ }^{3}$ The serum metabolic profile consisted of 1251 metabolites encompassing mainly lipids, amino acids, xenobiotics, carbohydrates, peptides, and nucleotides, and $30 \%$ of unidentified compounds. The reproducibility, accuracy, and long-term stability of the metabolomic data were validated by two tests. First, authors compared the levels of creatinine and cholesterol from metabolomics with those obtained using standardized laboratory tests, obtaining good correlation results (Pearson's $r>0.75$ ). Second, significant correlation (Spearman $p=0.68 \pm 0.06$ ) was obtained between metabolic profiles before and after one week in a small cohort. These results confirm that the metabolic profile is unique and specific for each individual. ${ }^{3}$

From the factors that affect the metabolome, diet and the gut microbiota had the largest predictive power with $48.9 \%$ and $30.8 \%$, respectively (Figure 1). This highlights and confirms the importance of diet and gut microbiota in the levels of serum metabolites. Robustness and reproducibility of the gut microbiota results were validated in two independent cohorts revealing strong associations between serum metabolites and gut microbiota. ${ }^{3}$ Interestingly, this relationship between the gut microbiota and serum in pediatric allergy and asthma has been recently described. ${ }^{4}$ Children with food allergy with or without asthma compared to those with asthma alone showed significant alterations in the metabolism of secondary biliary acids as well as aromatic amino acids. These are products

David Obeso and Elisa Zubeldia-Varela equally contributed.

(C) 2021 EAACl and John Wiley and Sons A/S. Published by John Wiley and Sons Ltd. 


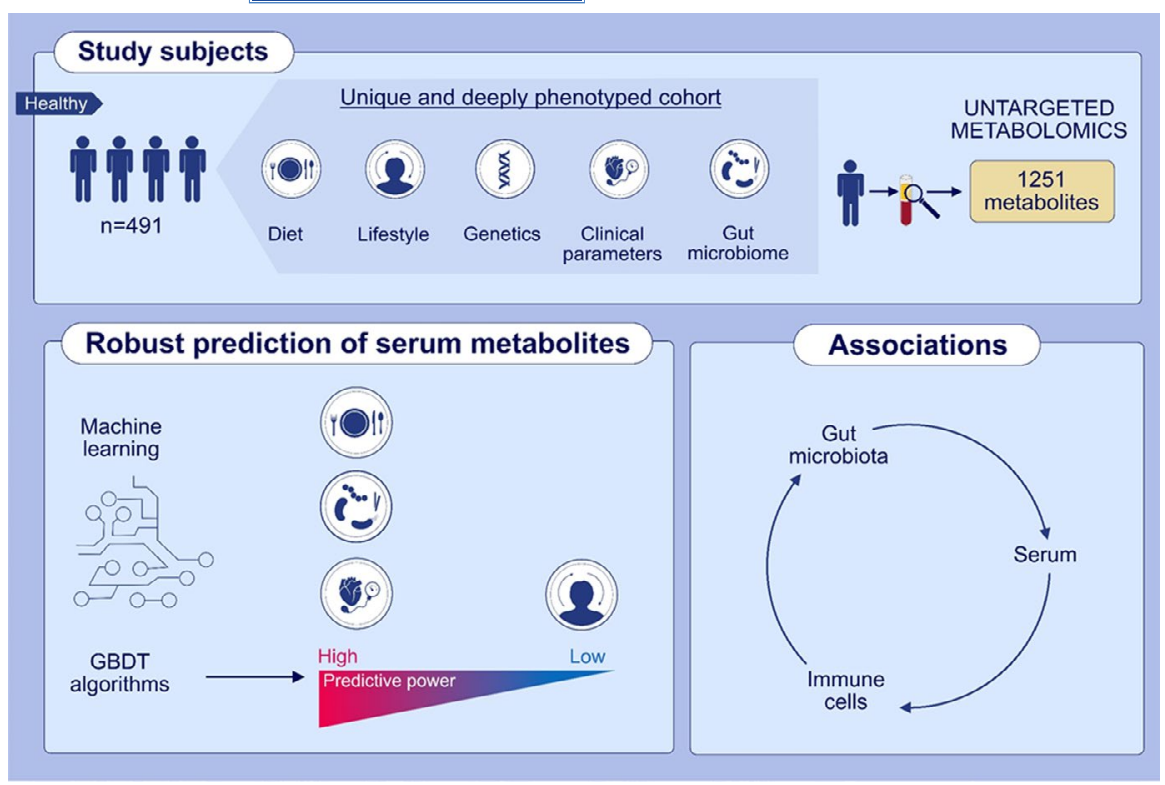

FIGURE 1 Influence of different factors on serum metabolites in health. The main factors in healthy individuals that affect serum metabolites are diet, lifestyle, genetics, clinical parameters and gut microbiota. Machine learning and gradient-boosted decision trees 12 (GBDT) showed that diet and the gut microbiota along clinical parameters are the highest predictive factors. There is a close relationship between intestinal microbiota, serum metabolites and immune cells

of microbiota-dependent enzymatic conversion in the gut. This study suggests that the observed modulation in amino acid and lipid metabolites may be strongly dependent on immune cells in terplay with the gut microbiota. ${ }^{4}$ Other metabolites related with gut microbiota are the short-chain fatty acids (SCFA). In a recent review, it was described that SCFAs acetate, propionate, butyrate, and pentanoate promote both regulatory $\mathrm{T}$ - and $\mathrm{B}$-cell differentiation and their potential beneficial effects on the prevention of food allergy. $^{5}$

Another review associates the gut microbiota with asthma and allergy and suggest that the mechanisms by which gut microbiota influence local immune responses include the altered differentiation of immune cell populations and the local production of metabolites that affect distal sites in asthma. ${ }^{6}$

Recent evidence in childhood atopic diseases using metabolomics suggest that tyrosine and tryptophan metabolism, lipids (e.g. sphingolipids), gut-derived metabolites, polyunsaturated fatty acids, and bile acids are the main involved pathways. ${ }^{7}$

Bar et al pointed that the gut microbiota may modulate the production of many circulating metabolites independent of diet. ${ }^{3}$ Among the taxa, Firmicutes and Bacteriodetes were the main bacterial predictors of serum metabolites. ${ }^{3}$ For instance, the ratio of the Firmicutes and Bacteroidetes phylum levels could be a significant indicator for certain diseases such as obesity.

Additionally, the modulation between diet and microbiota, and their impact on serum metabolome were tested. As a proof of concept Bar et al analyzed a week-long intervention with sourdough bread or industrial white bread. ${ }^{3}$ Indeed, changes in diet, and the intervention with prebiotics, probiotics, and symbiotics are different approaches to modulate the gut microbiota with the aim of treating diseases. These strategies, which are being increasingly studied, are promising for the development of a preventive therapy by restoring altered microbiome functionality or as an adjuvant in specific immunotherapy. ${ }^{8}$
Although the study of Bar et.al is compelling, it has some limitations. ${ }^{3}$ One of the main limitations is based on the use of samples from healthy individuals, as the disease and the pharmacological treatment are two of the main factors that modify the serum metabolome. As examples, current evidence has shown that severe allergic phenotypes displayed a characteristic plasma signature. ${ }^{9}$ In addition, grass-pollen sublingual immunotherapy response is different in serum of active and placebo allergic patients. ${ }^{10}$

To sum up, metabolomics is a useful resource for studies either for understanding the molecular mechanisms in health and disease, or in interventional studies with systemic metabolic alterations. The study by Bar et al fully demonstrates the influence of diet and gut microbiota in serum human metabolome.

\section{ACKNOWLEDGEMENT}

AV gratefully acknowledges the Martín-Carbajo family for supporting her scientific development through the X Mario Martín Velamazán award. We would like to thank Anna Globinska for her assistance in generating the graphical figure. Authors also acknowledge Professor Coral Barbas and Dr. Domingo Barber, for their asserted comments.

\section{CONFLICT OF INTEREST}

The authors have no conflict of interest. All authors have read and approved the manuscript.

\section{ORCID}

Alma Villaseñor (D) https://orcid.org/0000-0002-6652-2739

\section{REFERENCES}

1. Fiehn O. Metabolomics - the link between genotypes and phenotypes. Plant Mol Biol. 2002;48:155-171.

2. Falony G, Joossens M, Vieira-Silva S, et al. Population-level analysis of gut microbiome variation. Science 2016;352:560-564 
3. Bar N, Korem T, Weissbrod O, et al. A reference map of potential determinants for the human serum metabolome. Nature 2020;588:135. https://doi.org/10.1038/s41586-020-2896-2

4. Crestani E, Harb H, Charbonnier LM, et al. Untargeted metabolomic profiling identifies disease-specific signatures in food allergy and asthma. J Allergy Clin Immunol. 2020;145:897-906.

5. Luu M, Monning H, Visekruna A. Exploring the molecular mechanisms underlying the protective effects of microbial SCFAs on intestinal tolerance and food allergy. Front Immunol. 2020;11.

6. Ver Heul A, Planer J, Kau AL. The human microbiota and asthma. Clin Rev Allergy Immunol. 2019;57:350-363.

7. Schjødt MS, Gürdeniz G, Chawes B. The metabolomics of childhood atopic diseases: a comprehensive pathway-specific review. Metabolites. 2020;10:511.
8. Pascal M, Perez-Gordo M, Caballero T, et al. Microbiome and allergic diseases. Front Immunol. 2018;9.

9. Obeso D. Multi-omics analysis points to altered platelet functions in severe food-associated respiratory allergy. Allergy. 2018;73:2137-2149.

10. Barker-Tejeda TC. Exploring novel systemic biomarker approaches in grass-pollen sublingual immunotherapy using omics. Allergy. n/a.

How to cite this article: Obeso D, Zubeldia-Varela E, Villaseñor A. Uncovering the influence of diet and gut microbiota in human serum metabolome. Allergy. 2021;00:1-3. https://doi.org/10.1111/all.14770 